\title{
Enhanced ERK-1/2 activation in mice susceptible to coxsackievirus-induced myocarditis
}

\author{
Mary Anne Opavsky, ${ }_{1,2}$ Tami Martino, ${ }^{1}$ Marlene Rabinovitch, ${ }^{2}$ Josef Penninger, ${ }^{3}$ \\ Chris Richardson, ${ }^{3}$ Martin Petric, ${ }^{2}$ Cathy Trinidad, ${ }^{1,2}$ Lisa Butcher, ${ }^{1}$ Janice Chan, ${ }^{1}$ \\ and Peter P. Liu ${ }^{1}$
}

\begin{abstract}
${ }^{1}$ The Heart and Stroke/Richard Lewar Centre of Excellence, The University of Toronto, and The University Health Network, Toronto, Ontario, Canada

${ }^{2}$ The Hospital for Sick Children, Toronto, Ontario, Canada

${ }^{3}$ The Amgen Institute and the Departments of Immunology and Medical Biophysics, The University of Toronto, Ontario, Canada

Address correspondence to: Anne Opavsky, The Hospital for Sick Children, The Department of Pediatrics, Division of Infectious Diseases, 7313 Black Wing, 555 University Avenue, Toronto, Ontario, Canada, M5G 1X8.

Phone: (416) 813-6625; Fax: (416) 813-8404; E-mail: anne.opavsky@sickkids.ca.
\end{abstract}

Received for publication August 14, 2001, and accepted in revised form May 10, 2002.

Group B coxsackieviral (CVB) infection commonly causes viral myocarditis. Mice are protected from CVB3 myocarditis by gene-targeted knockout of $\mathrm{p} 56^{\mathrm{Lck}}(\mathrm{Lck})$, the Src family kinase (Src) essential for $\mathrm{T}$ cell activation. Extracellular signal-regulated kinase 1 and 2 (ERK-1/2) can influence cell function downstream of Lck. Using $\mathrm{T}$ cell lines and neonatal cardiac myocytes we investigated the role of ERK-1/2 in CVB3 infection. In Jurkat T cells ERK-1/2 is rapidly activated by CVB3; but, this response is absent in Lck-negative JCaM T cells. Inhibition of ERK-1/2 with UO126 reduced CVB3 titers in Jurkat cells, but not in JCaM cells. In cardiac myocytes CVB3 activation of ERK-1/2 is blocked by the Src inhibitor PP2. In addition, viral production in myocytes is decreased by Src or ERK-1/2 inhibition. In vitro, in both immune and myocardial cells, ERK-1/2 is activated by CVB3 downstream of Lck and other Src's and is necessary for efficient CVB3 replication. In vivo, following CVB3 infection, ERK-1/2 activation is evident in the myocardium. ERK-1/2 activation is intense in the hearts of myocarditis-susceptible A/J mice. In contrast, significantly less ERK- $1 / 2$ activation is found in the hearts of myocarditis-resistant C57BL/ 6 mice. Therefore, the ERK-1/2 response to CVB3 infection may contribute to differential host susceptibility to viral myocarditis.

J. Clin. Invest. 109:1561-1569 (2002). doi:10.1172/JCI200213971.

\section{Introduction}

Viral myocarditis is an important cause of acute and chronic heart failure (1). Group B Coxsackieviruses (CVBs), members of the Enterovirus genus, are the most common etiologic agents associated with acute viral myocarditis and chronic dilated cardiomyopathy $(1,2)$. The spectrum of disease spans from a fulminant course including arrhythmias or sudden death to mild symptoms and may later progress to chronic heart failure (1, 3 ). Investigations using murine models have demonstrated that both the T lymphocyte response to viral infection and direct CVB-mediated injury are important in the development of myocarditis following infection with CVB3 $(4,5)$. Gene-targeted knockout of both $\mathrm{CD}^{+}$ and $C D 8^{+}$T cells or the T cell receptor $\beta^{+}$(TCR- $\beta$ ) T cells results in minimal heart pathology following CVB3 infection (6). Because activation of p56 Lck (Lck), a member of the Src family $(\mathrm{Src})$ of protein tyrosine kinases, is required for normal $\mathrm{T}$ cell responses (7), we examined CVB3 infection in Lck-targeted knockout mice. In the absence of Lck, myocardial inflammation was completely absent, there was $100 \%$ survival of mice, and viral replication was impaired (8). The latter was confirmed by the significant diminution of CVB3 replication in
JCaM cells, which are Jurkat cells with no functional Lck (8). T cell stimulation by antigen or anti-TCR Ab's is characterized by a rise of intracellular tyrosine protein phosphorylation, which is dependent upon $\operatorname{Lck}(9,10)$. Downstream pathways include the mitogen-activated protein kinases (MAPKs). Important candidates are the extracellular signal-regulated kinases 1 and 2 (ERK-1/2), which are activated rapidly by MEK- $1 / 2$ through threonine and tyrosine phosphorylation following TCR engagement and Lck activation via adapter proteins and Ras $(11,12)$. ERK-1/2 are important participants in the regulation of cell proliferation and differentiation in response to a wide variety of growth factors and cytokines (13-15).

We have hypothesized that the ERK-1/2 signaling cascade is an important mediator of host susceptibility to CVB3 infection and disease. The role of ERK-1/2 was investigated in $\mathrm{T}$ cell lines and isolated neonatal cardiac myocytes, representative of the two cell types central to the pathogenesis of viral myocarditis. We find that in Jurkat $\mathrm{T}$ cells CVB3 infection specifically and rapidly activates the ERK-1/2 pathway in an Lckdependent manner. In addition, optimal CVB3 replication in Jurkat cells and cardiac myocytes is dependent 
upon activation of Src's and the ERK-1/2 signaling pathway. Furthermore, in CVB3-susceptible A/J mice we demonstrate that the ERK- $1 / 2$ signaling pathway was intensely activated early in the course of infection, associated with severe myocarditis and high cardiac viral titers. In contrast, in myocarditis-resistant C57BL $/ 6$ mice the ERK-1/2 response remains lower in association with less severe myocardial disease. Thus, our data support the concept that the ERK-1/2 cascade may be an important determinant of host susceptibility to CVB3 infection and virally induced myocarditis.

\section{Methods}

Antibodies and reagents. Phosphoprotein-specific mAbs detecting dual phosphorylated ERK-1/2, anti-ERK-1/2 $\mathrm{mAb}$, horseradish peroxidase-conjugated goat anti-rabbit (HRP-GAR) secondary Ab, and MEK-1 inhibitor PD98059 were purchased from New England Biolabs Inc. (Beverly, Massachusetts, USA). The MEK- $1 / 2$ inhibitor UO126 and the Src inhibitor PP2 were obtained from Calbiochem-Novabiochem Corp. (San Diego, California, USA). HRP-conjugated goat antimouse secondary (HRP-GAM) Ab was from DAKO Corp. (Carpinteria, California, USA). $\left[{ }^{35} \mathrm{~S}\right]$-methionine was supplied by Amersham Pharmacia Biotech (Baie d'Urfe, Quebec, Canada). All media, FCS, geneticin, and insulin were obtained from Life Technologies Inc. (Burlington, Ontario, Canada). Hygromycin B was obtained from Roche Diagnostics (Laval, Quebec, Cana$\mathrm{da}$ ), and 5-bromo-2-deoxyuridine (BRDU), transferrin, lithium chloride, selenium oxide, and ascorbic acid were from Sigma-Aldrich Canada Ltd. (Oakville, Ontario, Canada). The protein assay reagent was from Bio-Rad Laboratories Canada Ltd. (Mississauga, Ontario, Canada). An enhanced chemiluminescence detection system (ECL) from Amersham Pharmacia Biotech was used.

Cell culture. Jurkat T cells (E6-1), JCaM T cells, and TCR- $\beta$-negative (TCR- $\left.\beta^{-/}\right)$T cells were obtained from the American Type Culture Collection (ATTC) (Manassas, Virginia, USA). Cells were grown at $37^{\circ} \mathrm{C}$ with $5 \%$ $\mathrm{CO}_{2}$ in RPMI medium supplemented with $10 \mathrm{mM}$ HEPES, $1 \mathrm{mM}$ sodium pyruvate, $0.5 \%$ penicillin and streptomycin (P/S), and 10\% FCS. Jurkat/Lck cells and Jurkat/vector cells required additional supplementation with hygromycin B and geneticin.

Neonatal mouse cardiac myocyte cultures were prepared as described previously (16), with modifications. Briefly, hearts were removed from newborn C57BL/6 mice (Harlan Sprague-Dawley, Indianapolis, Indiana, USA) within 72 hours of birth. Following trimming, ventricles were mechanically minced and then subjected to stepwise enzymatic digestion with $0.15 \%$ trypsin. Isolated cells were washed with DMEM/HAM F-12 (1:1), pH 7.4, with $0.5 \% \mathrm{P} / \mathrm{S}$ (DMEM/F-12) plus $10 \%$ FCS. Nonmyocytes were depleted by a 90 -minute preplating on 10-cm2 Primaria tissue culture dishes (Becton-Dickinson, Franklin Lakes, New Jersey, USA). Myocytes were resuspended in media plus 10\% FCS with $0.1 \mathrm{mM}$ BRDU (to inhibit growth of nonmyocytes in the presence of serum) and plated in laminin-coated tissue-culture plates (Biocoat; Becton-Dickinson). After 24 to 48 hours, adherent myocytes were washed three times to remove serum and BRDU-containing media and resuspended in serum-free DMEM/F-12 plus the standard additives $(5 \mu \mathrm{g} / \mathrm{ml}$ transferrin, $1 \mathrm{nM}$ lithium chloride, $1 \mathrm{nM}$ selenium oxide, $25 \mu \mathrm{g} / \mathrm{ml}$ ascorbic acid, and $1 \mu \mathrm{g} / \mathrm{ml}$ insulin) to ensure optimal health of the myocytes. Insulin was excluded from the media in signaling experiments to minimize baseline ERK- $1 / 2$ activation. The myocytes were incubated an additional 24 hours before use.

Viruses. The cardiovirulent CVB3 strain CVB3-CG (CVB3) was adapted by Woodruff and Woodruff (5) and passaged in the laboratory of Charles Gauntt (Department of Microbiology, University of Texas Health Sciences Center at San Antonio, San Antonio, Texas, USA) (17). CVB3-VR30 (Nancy) was obtained from the ATCC. Stock viruses were prepared by passage once through HeLa cells. Virus was grown in HeLa cells until cytopathic effect was observed, then the cell monolayer was washed three times in serum-free RPMI and cells were collected in serum-free RPMI. Cells were lysed by freezing/thawing three times, clarified by centrifugation, and stored at $-80^{\circ} \mathrm{C}$, after titers were determined by plaque assay on HeLa cells. $\left[{ }^{35} \mathrm{~S}\right]$-methionine-labeled CVB3 ([$\left.\left.{ }^{35} \mathrm{~S}\right]-\mathrm{CVB} 3\right)$ was prepared in HeLa cell monolayers and purified by sucrose gradients as described previously (17).

To prepare purified virus for use in signaling experiments, clarified CVB3 and CVB3-VR30 stocks were separately overlaid on $15-45 \%(\mathrm{wt} / \mathrm{wt})$ sucrose gradients in collection buffer $(0.01 \mathrm{M} \mathrm{NaCl}, 0.01 \mathrm{M}$ Tris- $\mathrm{HCl}, 0.05 \mathrm{M}$ $\mathrm{MgCl}_{2}$ ). Gradients were then centrifuged at $100,000 \mathrm{~g}$ at $25^{\circ} \mathrm{C}$ for 3 hours in an SW28 rotor (Beckman Coulter Inc., Fullerton, California, USA). Prior to application to the gradients, some virus stocks were spiked with 1,000 $\mathrm{cpm}$ of radiolabeled virus. Following centrifugation, fractions with peak radioactivity or peak CVB3 titer were pooled and dialyzed in 10K Slide-A-Lyzer Cassettes (Pierce Chemical Co., Rockford, Illinois, USA) against RPMI medium. Virus titers of pooled and dialyzed peak fractions were determined by plaque assay, and aliquots were stored at $-80^{\circ} \mathrm{C}$.

CVB3 binding and replication. Binding assays of radiolabeled CVB3 were performed as described previously (17). Briefly, after washing in RPMI, cells $\left(5 \times 10^{6}\right.$ cells/test) were incubated with about $30,000 \mathrm{cpm}$ $\left[{ }^{35} \mathrm{~S}\right]$-CVB3 for 1 hour at room temperature (RT). Supernatant and two subsequent washes were collected and pooled (free virus) separately from the cell pellet (membrane-bound virus). The $\left[{ }^{35} \mathrm{~S}\right]-\mathrm{CVB} 3 \mathrm{cpm}$ were monitored by scintillation spectroscopy. The percentage of virus binding was calculated as follows: [cpm membrane-bound virus/(cpm membranebound + cpm-free virus)]

To determine viral production by $\mathrm{T}$ cell lines or cardiac myocytes, cells were washed with PBS, then adsorbed with CVB3 in serum-free media for 1 hour at RT. Cells 
were then washed to remove unbound virus, resuspended in RPMI plus 10\% FCS (T cell lines) or DMEM/F-12 plus standard additives (cardiac myocytes), and incubated at $37^{\circ} \mathrm{C}$. CVB3 titers were quantitated by plaque assay on HeLa cell monolayers.

CVB3 treatment of Jurkat cells and cardiac myocytes. Following incubation in RPMI with reduced serum $(0.5 \%$ FCS) for 18 hours, Jurkat or JCaM cell aliquots were washed in and resuspended in $200 \mu \mathrm{l}$ serum-free RPMI and preincubated at RT for 10 minutes. Sucrose-gradient purified CVB3 in serum-free RPMI or control serum-free RPMI was added to aliquots for the indicated times. Cells were incubated at RT for 5 to $10 \mathrm{~min}$ utes or at $37^{\circ} \mathrm{C}$ for 30 minutes or longer, and then cells were washed in PBS containing $400 \mu \mathrm{M}$ sodium orthovanadate, $5 \mathrm{mM}$ EDTA, and $10 \mathrm{mM}$ sodium fluoride. The cells were solubilized on ice for 15 minutes in lysis buffer containing $0.5 \%$ Triton X-100, $50 \mathrm{mM}$ Tris, $\mathrm{pH}$ 7.6, $300 \mathrm{mM} \mathrm{NaCl}, 1 \mathrm{mM}$ sodium orthovanadate, 5 $\mathrm{mM}$ EDTA, $10 \mu \mathrm{g} / \mathrm{ml}$ leupeptin, $10 \mu \mathrm{g} / \mathrm{ml}$ aprotinin, and $1 \mathrm{mM}$ PMSF. The lysates were spun at $12,000 \mathrm{~g}$ for 10 minutes at $4^{\circ} \mathrm{C}$, and protein concentrations of the supernatants were determined with the Bio-Rad Laboratories Canada Ltd. system.

Following incubation for 24 hours in serum-free, insulin-free DMEM/F-12 plus additives, monolayers of isolated neonatal cardiac myocytes were washed in PBS, then adsorbed with CVB3 in serum-free media for 1 hour at RT. Cells were then washed to remove unbound virus, resuspended in serum-free, insulin-free DMEM/F-12, and incubated at $37^{\circ} \mathrm{C}$ for 48 hours. Then, media was removed and monolayers were solubilized on ice for 15 minutes with lysis buffer.

Analysis of ERK-1/2 activation. Whole cell lysates were mixed with $2 \times$ Laemmli sample buffer, boiled, and proteins resolved by SDS-PAGE (8-16\% gradient gel; Novex, San Diego, California, USA) and transferred to PVDF. Membranes were blocked for 1 hour at RT with $5 \%$ powdered skim milk in TBS with $0.05 \%$ Tween (TBST), reacted with anti-phospho-ERK-1/2 $(1: 2,000)$ at $4^{\circ} \mathrm{C}$ overnight, and then incubated with HRP-GAM $\mathrm{Ab}(1: 1,000)$ for 1 hour at RT. Blots were developed with an ECL detection system. After membranes were incubated in stripping buffer $(10 \mathrm{mM} \beta$-mercaptoethanol, $2 \% \mathrm{wt} / \mathrm{vol}$ SDS, $62.5 \mathrm{mM}$ Tris, $\mathrm{pH}$ 6.7) for 30 minutes at $50^{\circ} \mathrm{C}$, they were washed in TBST, blocked, and incubated with anti-ERK-1/2 (1:1,000) at $4^{\circ} \mathrm{C}$ overnight. After incubation with HRP-GAR Ab $(1: 4,000)$ for 30 minutes at RT, total ERK-1/2 was detected with ECL. Ratios of phosphorylated/total ERK-1/2 were compared using densitometry (NIH Image Version 1.62; NIH, Bethesda, Maryland, USA).

CVB3 infection in mice. A/J (The Jackson Laboratories, Bar Harbor Maine, USA) and C57BL/6 mice (Harlan Sprague-Dawley), with documented differential susceptibility to CVB3 myocarditis (18), were inoculated intraperitoneally with $5 \times 10^{4}$ plaque-forming units (pfu) CVB3 at 6 weeks of age $(n=40$ per group). Animals were sacrificed on days 1,2 , and 4 after infection
( $n=5$ per group). Uninfected controls were sacrificed at day 0 . Organs were snap-frozen in liquid nitrogen and stored for later homogenization and tissue lysis, then processed as described above to detect ERK-1/2 activation. Alternatively, day- 4 hearts were embedded in OCT for cryosection. For the detection of infectious CVB3, homogenates of cardiac or splenic tissue were analyzed by plaque assay (6). Additional A/J and C57BL/6 mice were infected with $10^{4} \mathrm{pfu}$ CVB3 and followed to day 10 after infection to assess severity of myocarditis at the time of peak inflammation. Transverse cryosections (day 4) of paraffin-embedded sections (day 10) of hearts were stained with hematoxylin and eosin and examined histopathologically for evidence of inflammation and necrosis, as described previously (6).

\section{Results}

CVB3 infection of Jurkat cells activates the ERK-1/2 signaling pathway. T lymphocytes and $\mathrm{T}$ cell lines are known to be permissive to viral infection $(19,20)$ and are important in the pathogenesis of CVB3 myocarditis $(6,8)$. We used Jurkat cells, a human $T$ cell leukemia cell line, to examine the effects of CVB3 on intracellular signal transduction pathways. Specifically, to determine the effect of CVB3 on the ERK-1/2 signaling cascade, we first examined phosphorylation of the kinase in whole cell lysates collected from CVB3-treated and control Jurkat cells. Cardiovirulent CVB3 induced rapid ERK-1/2 phosphorylation as early as 5 minutes after treatment (Figure 1a). We have followed the phosphorylation status of ERK-1/2 for up to 4 hours after infection and have found that the response persists to 2 hours after infection (Figure 1b). In dose-response experiments CVB3 treatment at an moi of 25 was the threshold dose, with a moi of 50 consistently resulting in at least a two- to ninefold increase in ERK-1/2 phosphorylation. A minimal number or percentage of viral receptors may need to be bound by virus or cross-linked by the multivalent CVB3 to trigger subsequent signaling events. Activation of the ERK- $1 / 2$ cascade was also observed with a second strain of the virus, CVB3-VR30 (Nancy) (Figure 1c). These results indicate that CVB3 exposure triggers activation of the ERK-1/2 signaling in Jurkat cells.

CVB3-triggered ERK-1/2 activation in Jurkat cells is dependent on Lck. We reported previously that CVB3 replication is decreased in Lck knockout mice and also in JCaM cells, a Jurkat cell variant with no functional Lck (8). This led us to hypothesize that Lck controls host susceptibility to CVB3 infection via the ERK-1/2 signaling pathway. To explore a relationship between Lck and CVB3 activation of the ERK-1/2 signaling pathway, the intensity of ERK- $1 / 2$ phosphorylation was compared between infected Jurkat and JCaM cells. The dramatic activation of ERK-1/2 in Jurkat cells following exposure to CVB3 is in contrast to the essentially absent response in JCaM cells (Figure 2a). To determine if there may have been delayed activation in JCaM cells, incubation was continued for as long as 60 minutes before processing. This difference in phosphorylation 
a

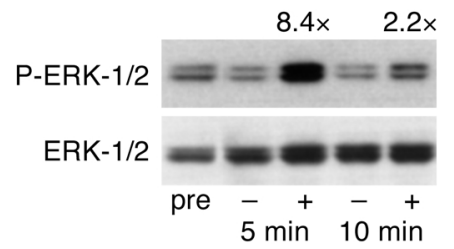

b

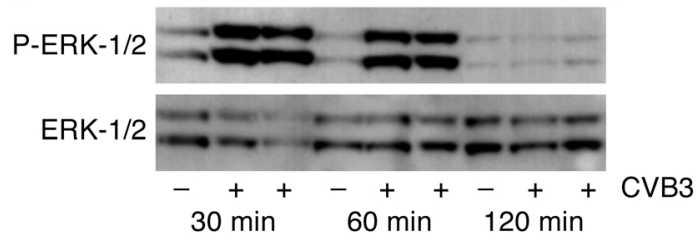

C

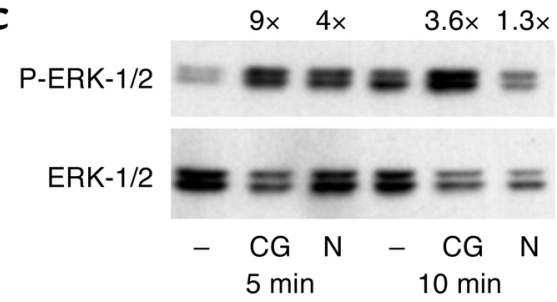

of ERK-1/2 was not due to a lack of MAPK expression because equivalent levels of total ERK- $1 / 2$ are present in both cell lines.

To confirm that Lck has an important role in viral activation of ERK-1/2, JCaM cells expressing Lck constitutively after transfection (JCaM/Lck) or controls transfected with vector alone (JCaM/vector) were exposed to CVB3. Treatment with CVB3 stimulated phosphorylation of ERK-1/2 by eightfold in Jurkat cells and ninefold in JCaM/Lck cells, in contrast to JCaM and JCaM/vector cells, which exhibited no phosphorylation of ERK-1/2 above baseline (Figure 2b). Treatment with the phorbol ester PMA rapidly activates the ERK- $1 / 2$ cascade in JCaM cells, indicating that the cell line does have an intact ERK- $1 / 2$ response system (not shown), despite low baseline levels of

\section{Figure 2}

Activation of the ERK-1/2 pathway by CVB3 infection is dependent on p56 Lck (Lck). (a) Jurkat cells or JCaM cells $\left(10^{7}\right)$ were incubated with CVB3 (moi $=50 ;+$ ) or control media (-) for 5 or 10 minutes, then lysed. One result represents three experiments. (b) Jurkat, $\mathrm{JCaM}$, and JCaM cells with control vector (JCaM/vector) or Lck restored $(\mathrm{JCaM} / \mathrm{Lck})\left(10^{7}\right)$ were incubated with CVB3 $(\mathrm{moi}=50 ;+)$ or control media (-) for 5 minutes, then lysed. One result represents two experiments. (c) TCR- $\beta$ chain negative Jurkat cells $\left(10^{7}\right)$ were lysed after incubation for 5 or 10 minutes with CVB3 $($ moi $=50 ;+)$ or control media (-), then lysed. One result represents two experiments. All cell lysates were immunoblotted with anti-phospho-ERK$1 / 2$ (P-ERK-1/2) and anti-total ERK-1/2 Ab's. Fold change in PERK-1/2-total ERK-1/2 ratio is indicated. (d) CVB3 binding to $T$ cell lines does not require activation of ERK-1/2. Jurkat and JCaM cells $\left(5 \times 10^{6}\right)$ were incubated with [ $\left.{ }^{35} \mathrm{~S}\right]$-CVB3 for 1 hour in the presence (PD98059) or absence (DMSO) of MEK-1/2 inhibitor. Binding of virus is expressed as mean percentages $( \pm$ SEM) for triplicate cultures. Viral binding to JCaM cells was twice as high as binding to Jurkat cells $\left({ }^{*} P=0.0001\right.$; Student $t$ test $)$.

\section{Figure 1}

CVB3 infection activates the ERK-1/2 pathway in Jurkat cells. (a) Jurkat cells $\left(10^{7}\right)$ were lysed before (pre) or after treatment with CVB3 ( $\mathrm{moi}=50 ;+)$ or control media $(-)$ for the indicated times. One result represents five experiments. (b) Jurkat cells $\left(10^{6}\right)$ were lysed 30 minutes to 4 hours after infection with CVB3 $($ moi $=50 ;+)$ or control media (-) for the indicated times. The increase in ERK-1/2 phosphorylation evident at 30 minutes to 2 hours after infection did not extend to the 4-hour time point. One result represents two experiments. (c) Five or 10 minutes after treatment with CVB3-CG (CG) or CVB3-VR30 $($ Nancy; N) (moi $=50)$, or control media $(-)$, cells $\left(10^{7}\right)$ were lysed. One result represents three experiments. All cell lysates were immunoblotted with anti-phospho-ERK-1/2 (P-ERK-1/2) and anti-total ERK-1/2 Ab's. Fold change in P-ERK-1/2-total ERK-1/2 ratio is indicated.

phosphorylation. We also treated TCR- $\beta^{-/}$Jurkat cells with CVB3 and observed ERK-1/2 phosphorylation, similar to wild-type Jurkat cells, indicating that a functional TCR, upstream of Lck, is not required for CVB3triggered activation of this MAPK (Figure 2c). These findings indicate that ERK-1/2 activation triggered by infection with CVB3 is dependent on the presence of Lck. Exposure to CVB3 did not alter activation of the p38 MAPK or SAPK/JNK cascades in either Jurkat or JCaM cells (not shown).

To investigate the importance of Lck and ERK- $1 / 2$ activation in viral attachment, as the first step in infection of cells, we measured binding of radiolabeled CVB3 to $T$ cell lines in the presence and absence of PD98059 (Figure 2d). We found that the absence of ERK-1/2 activation following viral infection in JCaM

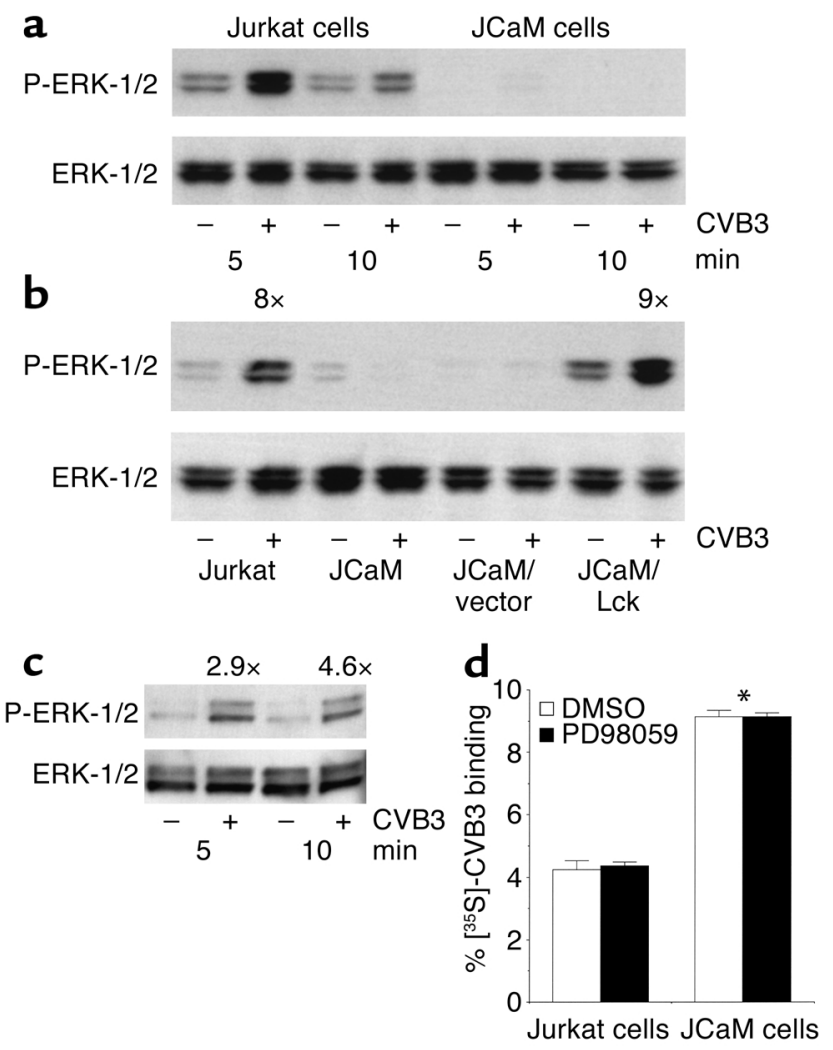


a

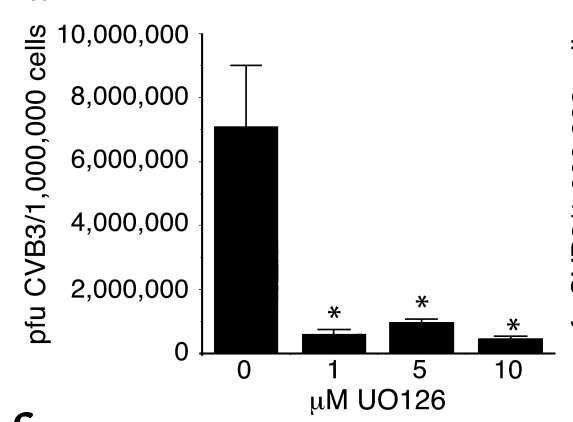

C
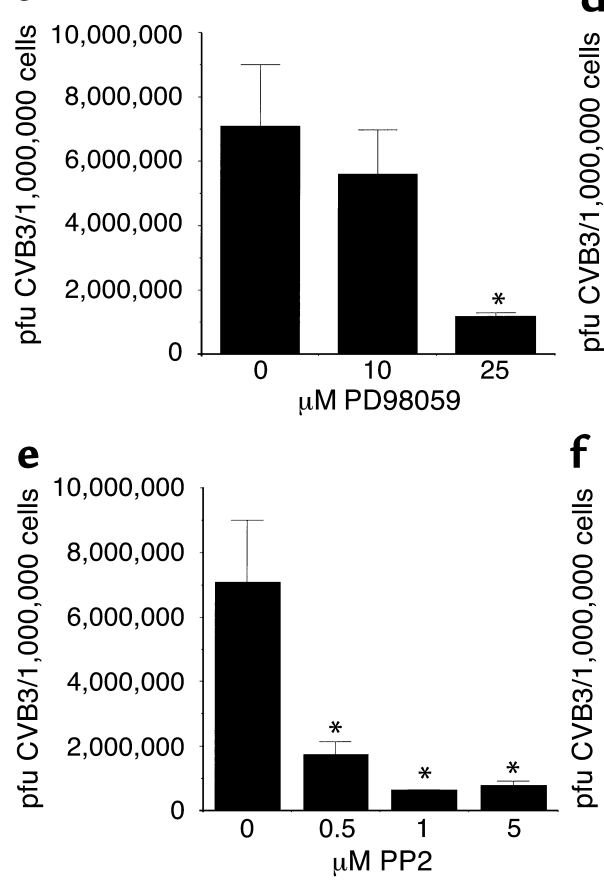

b

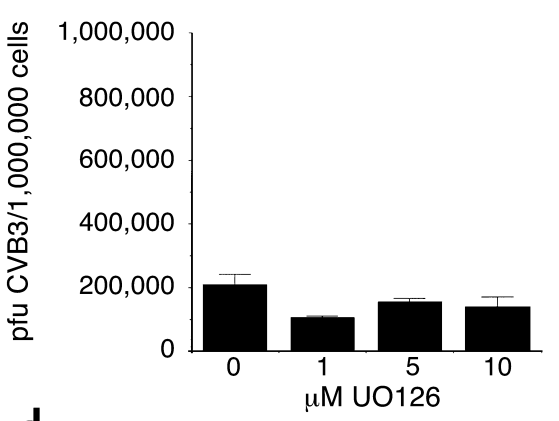

d

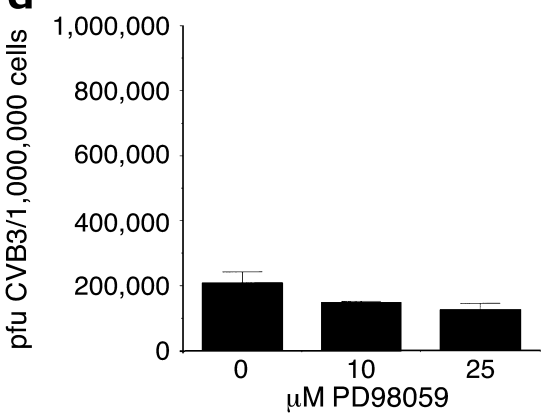

f

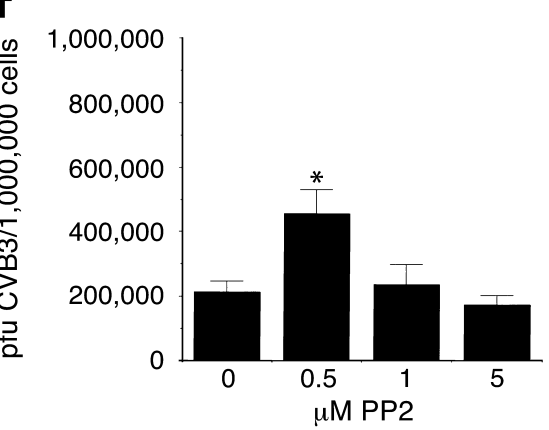

\section{Figure 3}

CVB3 replication in Jurkat cells is regulated by Src's and the ERK-1/2 signaling pathway. To assess infectivity, T cell lines $\left(10^{6}\right)$ were treated with kinase inhibitors in DMSO or with DMSO alone (0) for 1 hour at $37^{\circ} \mathrm{C}$, and then cells were infected with CVB3 ( $m o i=1)$ as described in Methods. Following incubation at $37^{\circ} \mathrm{C}$ for 48 hours, cells were frozen, then viral titers were determined by plaque assay. Treatment of (a) Jurkat and (b) JCaM cells with the MEK-1/2 inhibitor UO126 decreased CVB3 titers in Jurkat cells, but did not affect viral production in JCaM cells at the same doses of inhibitor. Treatment of (c) Jurkat and (d) JCaM cells with the MEK- $1 / 2$ inhibitor PD98059 significantly decreased CVB3 titers in Jurkat cells, but not in JCaM cells. Treatment of (e) Jurkat and (f) JCaM cells with the Src inhibitor PP2 decreased CVB3 titers in Jurkat cells, but not in JCaM cells. Titers were increased in JCaM cells treated with $0.5 \mu \mathrm{M}$ PP2. Virus titers are expressed as mean pfu $/ 10^{6}$ cells $( \pm \mathrm{SEM}, n=3$ per group). ${ }^{*} P<0.05$ DMSO versus kinase inhibitor for each cell type (ANOVA plus Bonferroni/Dunn post hoc testing). cells is not due to impaired CVB3 binding. In fact, despite decreased CVB3 yields in cells lacking Lck (JCaM), binding of $\left[{ }^{35} \mathrm{~S}\right]-\mathrm{CVB} 3$ was doubled in these cells, as compared with Jurkat cells. This may be due to a threefold increase in expression of the CVB receptor decay accelerating factor (DAF) in JCaM cells (not shown). Furthermore, MEK-1/2 inhibition did not reduce binding of radiolabeled CVB3 to either cell line, indicating that this initial attachment phase proceeds without ERK-1/2 activation.

CVB3 growth is dependent on activation of the ERK-1/2 pathway in Jurkat cells. The activation of ERK-1/2 observed following infection of Jurkat cells with CVB3 led us to investigate the importance of this signaling pathway in the viral replicative process. We found that the specific MEK-1/2 inhibitor UO126 reduced virus yield in Jurkat cells by up to $94 \%$ (Figure $3 a$ ). In contrast, treatment of Lck-negative JCaM cells with UO126 did not affect the already lower levels of CVB3 produced (Figure 3b). In addition, PD98059, also a MEK-1/2 inhibitor, decreased CVB3 titers in Jurkat cells by $83 \%$ (Figure 3c), but did not significantly affect viral production by JCaM cells at the same doses
(Figure $3 \mathrm{~d}$ ). These findings demonstrate a requirement for the activation of the ERK-1/2 signaling pathway for optimal CVB3 replication in Jurkat cells.

The Src (Lck, Hck, Fyn) inhibitor, PP2, decreased CVB3 production by Jurkat cells by $90 \%$ (Figure $3 \mathrm{e}$ ). This is consistent with the impaired replication in the Lck negative $T$ cell line. However, the lower baseline level of viral replication in JCaM cells was not inhibited by PP2 (Figure 3f). In fact, the lowest dose of PP2 increased CVB3 titers in JCaM cells versus control. So, while Lck is the primary Src required for efficient viral replication in $\mathrm{T}$ cells, other Src's may influence CVB3 infection as well.

Src and ERK-1/2 participate in the response of cardiac myocytes to CVB3 infection. We extended our investigation of the ERK-1/2 cascade in CVB3 infection to the heart, using isolated neonatal cardiac myocyte cultures. Treatment of cardiac myocytes with the MEK-1/2 inhibitor UO126 decreased the CVB3 titer significantly, as shown in Figure 4a. As well, the specific Src inhibitor, PP2, significantly suppressed CVB3 infection in cardiac myocytes (Figure 4b).

ERK-1/2 phosphorylation increased 2.6-fold 2 days after infection of cardiac myocytes with CVB3 (Figure 4c). 


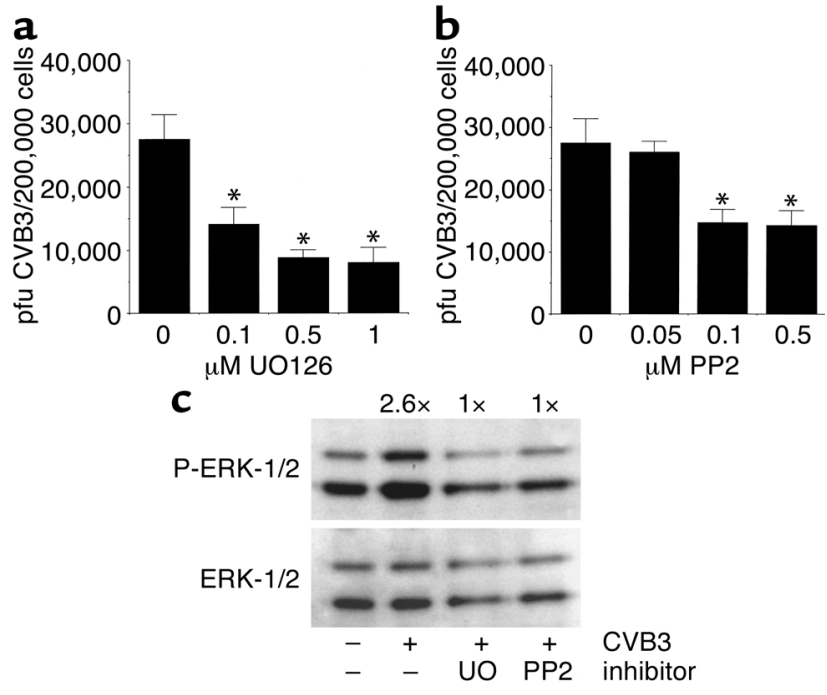

This activation of the ERK-1/2 pathway was inhibited by treatment with both MEK- $1 / 2$ and Src inhibitors, suggesting that these protein kinases regulate CVB3 infection of cardiac myocytes. Decreased CVB3 production by cardiac myocytes by MEK- $1 / 2$ and Src inhibitors and viral activation by ERK-1/2 are consistent with findings in the $\mathrm{T}$ cell model.

The relationship between ERK-1/2 activation and susceptibility to CVB3 myocarditis in mice. We observed that CVB3 activates ERK-1/2 in Jurkat cells and cardiac myocytes and that ERK-1/2 inhibition impairs viral growth in both of these cell types. We subsequently hypothesized that this signaling pathway may also be associated with host susceptibility to myocarditis in vivo. We proceeded to compare two mouse strains with differential susceptibility to myocarditis. CVB3 infection led to an acute and severe myocarditis in $\mathrm{A} / \mathrm{J}$ mice, but produced minimal myocardial inflammation and necrosis in C57BL/6 mice. Myocytolysis is evident in the myocardium of $\mathrm{A} / \mathrm{J}$ mice 4 days after infection, in the absence of any accompanying cellular infiltrate (Figure 5a). Cardiac myocytes are healthier and appear more intact in C57BL/6 mice at the same time point (Figure $5 \mathrm{~b}$ ). The peak inflammatory infiltrate

\section{Figure 5}

A/J mice are more susceptible to CVB3 myocarditis. At 4 days after infection representative cryosections $(10 \mu \mathrm{m})$ illustrate that (a) myocyte damage and myocytolysis (arrowheads) in the myocardium of $\mathrm{A} / \mathrm{J}$ mice are widespread, but (b) in the hearts of $\mathrm{C} 57 \mathrm{BL} / 6$ mice cardiac myocytes are generally intact, with cross-striations visible in normal-appearing myocytes. Ten days after infection, representative sections of paraffin-embedded tissue $(4 \mu \mathrm{m})$ illustrate (c) large areas of myocyte destruction and infiltrating mononuclear cells in the hearts of $\mathrm{A} / \mathrm{J}$ mice, but (d) only small isolated foci of inflammatory cells (arrowheads) in the myocardium of C57BL/6 mice. All histological sections were stained with hematoxylin and eosin, with original magnification $\times 400$. (e) CVB3 titers were significantly greater in A/J mouse hearts, consistent with the more aggressive heart disease evident on histopathology. ${ }^{*} P<0.05$ for $\mathrm{A} / \mathrm{J}$ mice day- 4 titers versus day- 1 and -2 titers; ${ }^{*} P<0.05$ for day- 4 titers in $\mathrm{A} / \mathrm{J}$ versus $\mathrm{C} 57 \mathrm{BL} / 6$ hearts ( $n=3$ per group; ANOVA plus Bonferroni/Dunn post hoc testing).

\section{Figure 4}

CVB3 replication in neonatal mouse cardiac myocyte cultures is regulated by Src's and the ERK-1/2 signaling pathway. (a) Cardiac myocytes, isolated from neonatal mice, were incubated with DMSO (0) or the MEK-1/2 inhibitor UO126 for 1 hour at $37^{\circ} \mathrm{C}$, infected with CVB3 ( $m o i=5$ ), and then incubated for 48 hours. (b) Cardiac myocytes were incubated with DMSO (0) or the Src's PP2 for 1 hour at $37^{\circ} \mathrm{C}$, then infected as above. Virus titers are expressed as mean pfu $/ 2 \times 10^{5}$ cells $\left( \pm\right.$ SEM, $n=3$ per group). ${ }^{*} P<0.05$ DMSO versus kinase inhibitor for each cell type (ANOVA plus Bonferroni/Dunn post hoc testing). (c) ERK-1/2 activation following CVB3 infection in isolated cardiac myocytes was blocked by inhibition of ERK-1/2 and Src activation. The increase in ERK-1/2 phosphorylation observed 48 hours after viral infection was inhibited by treatment with $1 \mu \mathrm{M} \cup \mathrm{O} 126$ (UO) and $1 \mu \mathrm{M}$ PP2. One result represents two experiments. Cell lysates were immunoblotted with anti-phosphoERK-1/2 (P-ERK-1/2) and anti-total ERK-1/2 Ab's. Fold change in P-ERK-1/2-total ERK-1/2 ratio is indicated.

in the myocardium occurs at about day 10 in this model. Large areas of myocyte destruction and infiltrating mononuclear cells are found in the hearts of $\mathrm{A} / \mathrm{J}$ mice 10 days following CVB3 infection; however, myocardial disease is minimal in C57BL/6 mice (Figure 5, $\mathrm{c}$ and d). Cardiac viral titers were initially similar between the two strains, but by day 4 viral titers were significantly higher in the hearts of $\mathrm{A} / \mathrm{J}$ mice compared with C57BL/6 mice (Figure 5e). In contrast, peak viral titers in the spleen were not different between the two strains of mice (not shown).
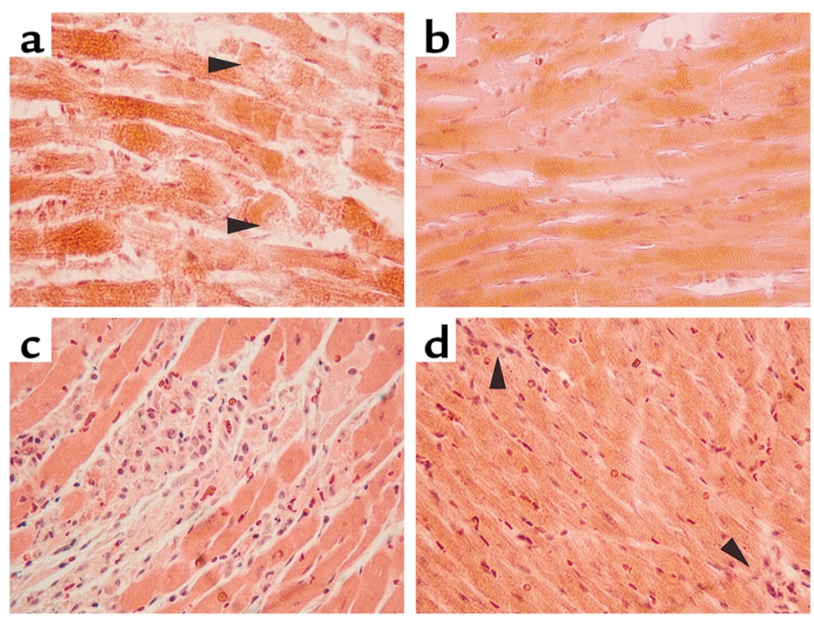

e

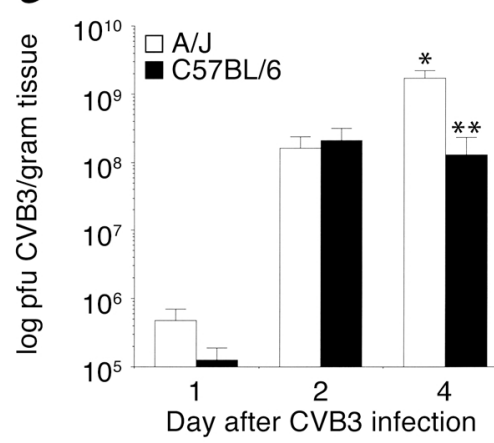



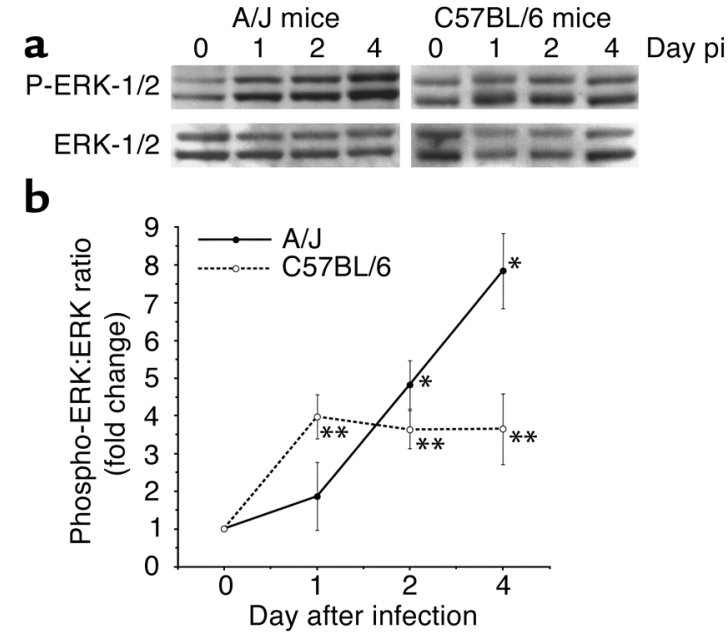

\section{Figure 6}

ERK-1/2 activation in the heart is associated with susceptibility to CVB3 myocarditis. (a) ERK-1/2 phosphorylation was increased in the hearts of CVB3-infected mice as early as day 1 postinfection (pi). By day 4, ERK-1/2 activation was more intense in myocarditis-susceptible $A / J$ mice. Levels of phosphorylated ERK-1/2 (P-ERK-1/2) and total ERK-1/2 in the heart were determined by Western blot analysis of tissue homogenates from uninfected controls $(0)$ and days 1,2 , and 4 after CVB3 infection. Results shown are representative of 5 mice per group. (b) The fold change in myocardial P-ERK-1/2:total ERK $-1 / 2$ ratios from day 0 (baseline set at 1 ) was determined following densitometry. By day 2 after infection, ERK-1/2 activation in the myocardium of $\mathrm{A} / \mathrm{J}$ mice was elevated fivefold above the baseline in uninfected control mice. In A/J mice the intensity of ERK-1/2 phosphorylation continued to increase to eight times baseline by day 4 after infection. ERK-1/2 activation persisted at a lower level in the hearts of myocarditis-resistant C57BL/ 6 mice. Values are expressed as the mean fold change from baseline ( $\pm \mathrm{SEM} ; n=5$ per group). ${ }^{*} P<0.05$ versus day 0 for $\mathrm{A} / \mathrm{J}$ mice, and on day $4 \mathrm{~A} / \mathrm{J}$ versus $\mathrm{C} 57 \mathrm{BL} / 6$ mice; ${ }^{*} P<0.05$ versus day 0 for $\mathrm{C} 57 \mathrm{BL} / 6$ mice (ANOVA plus Bonferroni/Dunn post hoc testing).

ERK-1/2 phosphorylation in the myocardium of CVB3-infected $\mathrm{A} / \mathrm{J}$ mice increased in parallel with high viral titers and severity of myocarditis. ERK- $1 / 2$ is activated as early as 24 hours after infection, before peak viral titers are reached (Figure 6a). By day 2 after CVB3 infection of $\mathrm{A} / \mathrm{J}$ mice, ERK- $1 / 2$ activation in the myocardium was elevated fivefold above the baseline in uninfected control mice. In $\mathrm{A} / \mathrm{J}$ mice the intensity of ERK-1/2 phosphorylation continued to increase to eightfold baseline by day 4 following infection (Figure 6 , a and b). ERK-1/2 activation persisted at a significantly lower level in the hearts of myocarditis-resistant $\mathrm{C} 57 \mathrm{BL} / 6$ mice. In the spleens no significant change in ERK-1/2 activation was observed (data not shown).

\section{Discussion}

We have presented evidence in this study in support of our hypothesis that activation of the ERK-1/2 signaling cascade is a potential determinant of susceptibility to CVB3 infection. To gain insight into the role of ERK-1/2 in the pathogenesis of CVB3 infection we studied both $\mathrm{T}$ cells and cardiac myocytes. We have found that CVB3 infection triggers activation of the ERK-1/2 signaling cascade and that optimal viral replication depends on ERK-1/2 activation. Virus appears to amplify its own replication by enlisting the host cell's signal transduction system, specifically, the ERK-1/2 pathway. The data suggest a Src-dependent activation of ERK- $1 / 2$ by CVB3 is common to both $\mathrm{T}$ cells and cardiac myocytes and may be significant in the whole animal's response to infection. This concept is supported in a mouse model of host susceptibility to myocarditis. Intense phosphorylation of ERK- $1 / 2$ in the myocardium is specifically correlated with high cardiac titers of CVB3 and severe myocarditis. ERK-1/2 activation persists at lower levels in the hearts of mice with relative resistance to CVB3 myocarditis.

Following gene-targeted knockout of Lck, mice are protected from CVB3 myocarditis (8). Our studies in $T$ cell lines suggest that one mechanism by which Lck may influence the myocarditis phenotype is via activation of the ERK-1/2 pathway. Experiments described here demonstrate that CVB3 stimulates rapid phosphorylation of ERK-1/2 in an Lck-dependent manner. In addition, viral production depends on activation of the ERK-1/2 cascade in Jurkat cells. However, Src or ERK-1/2 inhibition does not significantly decrease the already lower level of viral production in JCaM cells. These data suggest that Lck is upstream of ERK-1/2 and is the primary Src required for efficient CVB3 replication in $\mathrm{T}$ cells. In cardiac myocytes CVB3 also activates ERK-1/2 in a Srcdependent manner. As with Jurkat cells, ERK-1/2 activation is required for peak susceptibility to viral infection. Thus, the ERK- $1 / 2$ signaling cascade is not only activated by CVB3, but is a host cell component essential to productive replication of the virus. This leads to the hypothesis of a self-amplification process, whereby the virus promotes its own growth by activation of the ERK- $1 / 2$ cascade.

Findings thus far suggest that Src's in cardiac myocytes may have a comparable role to Lck in the $T$ cell. In mice lacking the Lck gene, viral titers in the heart were decreased 4 days after CVB3 infection (8). This is consistent with the decrease in viral production by isolated cardiac myocytes with inhibition of Src's. Interestingly, we have detected Lck RNA using RT-PCR and Lck protein in the hearts of both $\mathrm{A} / \mathrm{J}$ and $\mathrm{C} 57 \mathrm{BL} / 6$ mice (not shown). In addition, several Src's, including Lck, are expressed in the rabbit heart. In the adult rabbit and isolated cardiac myocytes Lck is activated by ischemic preconditioning, an established cardioprotective technique (21). These observations challenge the traditional concept that Lck is expressed only in $\mathrm{T}$ cells, B cells, and natural killer cells (22). While Lck may be a primary upstream kinase that influences ERK-1/2 phosphorylation in response to CVB3 in $T$ cells, the specific contribution of other protein kinases to regulation of the viral life cycle remains to be determined. Interestingly, in Lck-negative JCaM cells, treatment with a low dose of the Src inhibitor PP2 
increased CVB3 titers, while higher doses of PP2 had no effect. While Lck appears to be required for optimal viral replication, perhaps other Src's inhibit viral replication in the absence of Lck. The dose-dependent effect of PP2 on viral replication may reflect differential inhibition of Src's. Alternative mechanisms for ERK-1/2 activation, other than through Lck or related Src's, may also influence the viral replicative process and the host cell response to CVB3 infection at different times following infection.

Several viruses have evolved diverse mechanisms to stimulate signal transduction pathways that may promote viral replication, regulate host inflammatory responses to infection, or induce viral oncogenic transformation of cells. Reports of the effects of Enteroviruses on intracellular signal transduction pathways are limited and focus on later stages of infection, at the time of virus-induced host protein synthesis shutoff (23-25). During infection with echovirus 1 and 7, ERK-1/2 activation was shown to be induced by 5 hours after infection, while p38 MAPK was activated by 10 hours after infection (25). CVB3 infection has been shown to induce tyrosine phosphorylation of host proteins in HeLa cells from 3 to 5 hours after infection (23). Sam68, a cellular target of Src's, interacts with the poliovirus RNAdependent RNA polymerase 3D (26) and associates with RasGAP in response to CVB3 infection (24). RasGAP is cleaved at 6 hours after infection, possibly activating the Ras/MAPK pathway (24). The authors hypothesized that the persistent ERK-1/2 activation observed at this late stage of infection may affect CVB3-induced cytotoxicity or apoptosis. In contrast, by focusing earlier in the viral life cycle, we have demonstrated a requirement for this growth-activated MAPK in efficient CVB3 replication. The potential for a multiplicity of roles for protein kinases in viral infection has been illustrated by the study of signal transduction pathways involved in HIV infection. A Lck activation in HIV-mediated signal transduction is consistent with viral binding to $\mathrm{CD} 4$, the major HIV receptor (27). As well, HIV-1 binding to CD4 receptors activates the MEK/ERK signal transduction pathway (28). In addition, ERK- $1 / 2$ enhances the infectivity of HIV-1 virions themselves, in part by phosphorylation of the viral proteins Vif, Rev, Tat, $\mathrm{Nef}$, and p17 ${ }^{\mathrm{Gag}}$ (29). In CVB3-infected cells the downstream effects of ERK-1/2 activation may influence both the function of host cellular proteins and the infectivity of viral proteins, as with HIV-1. Activation of the ERK-1/2 cascade may influence CVB3 replication at any time from viral entry to progeny release. Viral binding appears to proceed independently of ERK-1/2 activation; however, early steps, such as viral entry or uncoating may be dependent on activation of this kinase in the first 2 hours following infection. It possible that regulation of early events in the viral life cycle may have an impact downstream, affecting total viral yield.
In the hearts of infected mice ERK- $1 / 2$ is activated as early as 24 hours following CVB 3 inoculation, before peak cardiac viral titers are reached. Interestingly, ERK- $1 / 2$ activation is not transient. Perhaps this is due to the presence of ongoing viral stimulation, as it replicates and spreads through the myocardium. Consistent with this is the ERK-1/2 activation present in isolated cardiac myocytes 48 hours following CVB3 infection. ERK-1/2 phosphorylation increases from day 0 to day 4 in the hearts of susceptible A/J mice. In contrast, increased ERK-1/2 phosphorylation persists at a lower level in the hearts of myocarditis-resistant C57BL/6 mice. The higher peak cardiac viral titers in susceptible A/J mice 4 days after infection may stimulate the increased levels of ERK-1/2. Early ERK-1/2 activation may also promote viral replication, to differing degrees, depending on the mouse strain. In vitro observations support both CVB3 activation of ERK- $1 / 2$ and the influence of ERK-1/2 on viral production. The absence of inflammatory foci in the myocardium of mice at day 4 after infection, particularly in comparison with infiltrates seen at day 10 , suggest that the source of phosphorylated ERK-1/2 is not infiltrating immune cells.

In summary, we have demonstrated that the ERK-1/2 signaling cascade is a potentially novel determinant of susceptibility to CVB3 infection and disease. In T cell lines the ERK- $1 / 2$ pathway is specifically and rapidly activated in an Lck-dependent manner. This supports ERK-1/2 activation as a mechanism by which Lck controls susceptibility to CVB3 myocarditis. CVB3 may in this way promote the exuberant $\mathrm{T}$ lymphocyte response found in CVB3 myocarditis. As demonstrated by the infection of murine cardiac myocytes with CVB3, the importance of Src and the ERK-1/2 cascade in the establishment of viral infection is not restricted to $\mathrm{T}$ cells. The virus may take advantage of one element of the host's signal transduction system to promote its own infectivity. Consistent with observations made in vitro in T cells and cardiac myocytes, activation of the ERK-1/2 pathway in the heart is associated with elevated CVB3 replication and pathogenicity in mice. Moreover, ERK-1/2 activation is increased in the hearts of mice susceptible to severe myocarditis. The discovery of different patterns of ERK- $1 / 2$ activation in the hearts of myocarditis-susceptible and -resistant mice highlights a critical juncture downstream of viral receptors, which, with further study, may lead to new strategies in the treatment of severe CVB disease.

\section{Acknowledgments}

We greatly appreciate the technical assistance of Fayez Dawood and Wen-Hu Wen with the animal protocols. This research was supported by grants from the Canadian Institutes of Health Research (CIHR) and the Heart and Stroke Foundation of Ontario. M.A. Opavsky was a postdoctoral fellow of the CIHR. P.P. Liu is the Heart and Stroke/Polo Endowed Research Chair at the University of Toronto. 
1. Opavsky, M.A., Sole, M.J., and Liu, P. 1998. Myocarditis. In Cardiology. W Parmley and K. Chatterjee, editors. Lippincott-Raven. Philadelphia, Pennsylvania, USA.

2. Woodruff, J. 1980. Viral myocarditis. A review. Am. J. Pathol. 101:427-484.

3. Liu, P., Martino, T., Opavsky, M.A., and Penninger, J. 1996. Viral myocarditis: balance between viral infection and immune response. Can. J. Cardiol. 12:935-943.

4. Chow, L.H., Beisel, K.W., and McManus, B.M. 1992. Enteroviral infection of mice with severe combined immunodeficiency: evidence for direct viral pathogenesis of myocardial injury. Lab. Invest. 66:24-31.

5. Woodruff, J.F., and Woodruff, J.J. 1974. Involvement of T lymphocytes in the pathogenesis of Coxsackie virus B3 heart disease. J. Immunol. 113:1726-1734.

6. Opavsky, M.A., et al. 1999. Susceptibility to myocarditis is dependent on the response of $\alpha \beta$ T lymphocytes to coxsackieviral infection. Circ. Res. 85:551-558.

7. Voronova, A.F., Buss, J.E., Patschinsky, T., Hunter, T., and Sefton, B.M. 1984 Characterization of the protein apparently responsible for the elevated tyrosine protein kinase activity in LSTRA cells. Mol. Cell Biol. 4:2705-2713.

8. Liu, P., et al. 2000. The tyrosine kinase p56lck is essential in Coxsackievirus B3-mediated heart disease. Nat. Med. 6:429-434.

9. Hsi, E.D., et al. 1989. T cell activation induces rapid tyrosine phosphorylation of a limited number of cellular substrates. J. Biol. Chem. 264:10836-10842.

10. Straus, D.B., and Weiss, A. 1992. Genetic evidence for the involvement of the Lck tyrosine kinase in signal transduction through the T cell antigen receptor. Cell. 70:585-593.

11. Franklin, R., et al. 1994. Ligation of the $\mathrm{T}$ cell receptor complex results in activation of the Ras/Raf-1/MEK/MAPK cascade in human T lymphocytes. J. Clin. Invest. 93:2134-2140.

12. Gutkind, J.S. 1998. The pathways connecting $G$ protein-coupled receptors to the nucleus through divergent mitogen-activated protein kinase cascades. J. Biol. Chem. 273:1839-1842.

13. Sturgill, T.W., Ray, L.B., Erikson, E., and Maller, J.L. 1988. Insulin-stimulated MAP-2 kinase phosphorylates and activates ribosomal proteins S6 kinase II. Nature. 334:715-718.

14. Boulton, T.G., et al. 1991. ERKs: a family of protein-serine/threonine kinases that are activated and tyrosine phosphorylated in response to insulin and NGF. Cell. 65:663-675.

15. Davis, R.J. 1993. The mitogen-activated protein kinase signal transduction pathway. J. Biol. Chem. 268:14553-14556.

16. Parker, T.G., Packer, S.E., and Schneider, M.D. 1990. Peptide growth fac- tors can provoke "fetal" contractile protein gene expression in rat cardiac myocytes. J. Clin. Invest. 85:507-514.

17. Martino, T.A., et al. 1998. Cardiovirulent Coxsackieviruses and the decay-accelerating factor (CD55) receptor. Virology. 244:302-314

18. Chow, L., Gauntt, C., and McManus, B. 1991. Differential effects of myocarditic variants of Coxsackievirus B3 in inbred mice: a pathologic characterization of heart tissue damage. Lab. Invest. 64:55-64.

19. Vuorinen, T., Vainionpaa, R., Kettinen, H., and Hyypia, T. 1994. Coxsackievirus B3 infection in human leukocytes and lymphoid cell lines. Blood. 84:823-829.

20. Vuorinen, T., Vainionpaa, R., Vanharanta, R., and Hyypia, T. 1996. Susceptibility of human bone marrow cells and hematopoietic cell lines to Coxsackievirus B3 infection. J. Virol. 70:9018-9023.

21. Ping, P., et al. 1999. Demonstration of selective protein kinase C-dependent activation of Src and Lck tyrosine kinases during ischemic preconditioning in conscious rabbits. Circ. Res. 85:542-550.

22. Bolen, J.B., Rowley, R.B., Spana, C., and Tsygankov, A.Y. 1992. The Src family of tyrosine protein kinases in hemopoietic signal transduction. FASEB J. 6:3403-3409.

23. Huber, M., Selinka, H.-C., and Kandolf, R. 1997. Tyrosine phosphorylation events during Coxsackievirus B3 replication. J. Virol. 71:595-600.

24. Huber, M., et al. 1999. Cleavage of RasGAP and phosphorylation of mitogen-activated protein kinase in the course of Coxsackievirus B3 replication. J. Virol. 73:3587-3594.

25. Huttunen, P., Hyypia, T., Vihinen, P., Nissinen, L., and Heino, J. 1998. Echovirus 1 infection induces both stress- and growth-activated mitogen-activated protein kinase pathways and regulates the transcription of cellular immediate-early genes. Virology. 250:85-93.

26. McBride, A., Schlegel, A., and Kirkegaard, K. 1996. Human protein Sam 68 relocalization and interaction with poliovirus RNA polymerase in infected cells. Proc. Natl. Acad. Sci. USA. 93:2296-2301.

27. Manna, S.K., and Aggarwal, B.B. 2000. Differential requirement for p56lck in HIV-tat versus TNF-induced cellular responses: effects on $\mathrm{NF}-\mathrm{kB}$, activator protein-1, c-jun N-terminal kinase, and apoptosis. J. Immunol. 164:5156-5166.

28. Popik, W., Hesselgesser, J.E., and Pitha, P.M. 1998. Binding of human immunodeficiency virus type 1 to CD4 and CXCR 4 receptors differentially regulates expression of inflammatory genes and activates the MEK/ERK signaling pathway. J. Virol. 72:6406-6413.

29. Yang, X., and Gabuzda, D. 1999. Regulation of human immunodeficiency virus type 1 infectivity by the ERK mitogen-activated protein kinase signaling pathway. J. Virol. 73:3460-3466. 